\title{
Developmental toxicity and anti- inflammatory effect of the soft coral Dendronephthya gigantea collected from Jeju Island in zebrafish model
}

\author{
Seung-Hong Lee
}

\begin{abstract}
Recent in vitro studies have demonstrated that extract of soft coral Dendronephthya gigantea (SCDE) had strong anti-inflammatory activities. However, the direct effects of SCDE on anti-inflammatory activities in vivo model remained to be determined. Therefore, the present study was designed to assess in vivo anti-inflammatory effect of SCDE using lipopolysaccharide (LPS)-stimulated zebrafish model. We also investigated whether SCDE has toxic effects in zebrafish model. The survival, heart beat rate, and developmental abnormalities were no significant change in the zebrafish embryos exposed to at a concentration below $100 \mu \mathrm{g} / \mathrm{ml}$ of SCDE. However, lethal toxicity was caused after exposure to 200 and $400 \mu \mathrm{g} / \mathrm{ml}$ of SCDE. Treating zebrafish model with LPS treatment significantly increased the reactive oxygen species (ROS) and nitric oxide (NO) generation. However, SCDE inhibited this LPS-stimulated ROS and NO generation in a dose-dependent manner. These results show that SCDE alleviated inflammation by inhibiting the ROS and NO generation induced by LPS treatment. In addition, SCDE has a protective effect against the cell damage induced by LPS exposure in zebrafish embryos. This outcome could explain the profound anti-inflammatory effect of SCDE both in vitro as well as in vivo, suggesting that the SCDE might be a strong anti-inflammatory agent.
\end{abstract}

Keywords: Soft coral, Dendronephthya gigantea, Anti-inflammatory effect, Toxicity, Zebrafish model

\section{Background}

Many marine organisms have unique structures that are not found in terrestrial organisms. Soft corals are a group of colonial invertebrates which form a significant set of marine organisms occurring widely in the coral reefs throughout the world (Lakshmi and Kumar 2009; Blunt et al. 2012). Among the Alcyonacean soft corals, genus Dendronephthya is one of the most widely distributed soft corals genera throughout tropical coastal water of the Indian Ocean, Pacific Ocean, and Southeast Asia (Elkhayat et al. 2014). Dendronephthya species have been recognized as rich sources of novel and diverse chemical structures with interesting biological activities (Tomono et al. 1999; Li et al. 2005; Chao et al. 2008).

Correspondence: seunghong0815@gmail.com

Department of Pharmaceutical Engineering, Soonchunhyang University, Asan 31538, Republic of Korea
Jeju Island, Korea's southernmost island, is geographically affected by the Taiwan warm current; as a consequence, it becomes a place where temperate and subtropical creatures coexist, with a higher diversity of species and unique formation of marine biota (Cho et al. 2014). Recently, the number of Alcyonacean soft coral populations in the sea of Jeju Island is increasing due to the tropical weather. Recent in vitro studies have demonstrated that extract of Dendronephthya gigantea collected from Jeju Island possesses anti-inflammatory effect (Wang et al. 2016). Although such results indicate the potential use of the soft coral D. gigantea extract (SCDE) as anti-inflammatory candidates, no studies have yet reported its effects on toxicity and anti-inflammatory activities in vivo model.

The vertebrate zebrafish (Danio rerio) is a small tropical freshwater fish, which has emerged as a useful vertebrate model organism because of its small size, large 
clutches, transparency, low-cost maintenance, and morphological and physiological similarity to mammals (Eisen 1996; Fishman 1999). Due to these advantages, recently, the value of the zebrafish as a model organism for in vivo drug toxicity and efficacy studies has been recognized (den Hertog 2005; Ali et al. 2011; He et al. 2013). In addition, the optical transparency of zebrafish embryos allows noninvasive and dynamic imaging the inflammation in vivo. Therefore, zebrafish are a useful and popular animal model for a variety of inflammation studies. In in vivo anti-inflammation test model, zebrafish is widely accepted as the best method for effective anti-inflammation assay (Liao et al. 2011; Park and Cho 2011; Lee et al. 2013). Therefore, the purpose of this study was to evaluate the developmental toxicity of SCDE and to examine its in vivo anti-inflammatory effect in zebrafish model.

\section{Methods}

\section{Preparation of the soft coral Dendronephthya gigantea} extract (SCDE)

Soft coral D. gigantea were collected from the coast of the Jeju Island, Korea, and were identified by Jeju Biodiversity Research Institute, Jeju Technopark. After surface disinfection with $70 \%$ ethanol spray to kill the surface-attached microorganisms, the samples were washed with tap water to remove any surface attachments and debris. Sample was then lyophilized and ground to form a powder. Each $20 \mathrm{~g}$ sample of the soft coral lyophilized powder underwent extraction using 21 of $70 \%$ ethanol at $25{ }^{\circ} \mathrm{C}$ for $24 \mathrm{~h}$. Extraction was carried out three times for each sample. Finally, the filtered extracts were concentrated using a rotary evaporator under vacuum.

\section{Origin and maintenance of parental zebrafish}

Ten adult zebrafish were obtained from a commercial dealer (Seoul aquarium, Seoul, Korea) and were kept in a 3-1 acrylic tank at $28.5{ }^{\circ} \mathrm{C}$ with a $14: 10$-h light:dark cycle. The zebrafish were fed three times a day, 6 days/ week, with tetramin flake food supplemented with live brine shrimps (Artemia salina; SEWHAPET food Co., Seoul, Korea). Embryos were obtained from natural spawning that was induced in the morning by turning on the light. The collection of embryos was completed within $30 \mathrm{~min}$ in petri dishes.

\section{Measurement of embryo toxicity}

Zebrafish embryos at 7-9 h post-fertilization (hpf) were randomly distributed in 12-well plates at a density of 10 embryos/well, containing $2 \mathrm{ml}$ of embryo medium per well. The embryos were treated with various concentrations of SCDE for $72 \mathrm{~h}$ at concentrations of $1,10,100$, 200 , and $400 \mu \mathrm{g} / \mathrm{ml}$. Final DMSO concentrations were
$0.1 \%$ in the treatment solution, and $0.1 \%$ DMSO was used as vehicle control during the assays. Medium was not renewed throughout the experiment.

Survival rate was monitored daily during the whole experiment. Any dead embryos were removed every day until $72 \mathrm{hpf}$. For cardiac toxicity measurement, embryos were anesthetized in $0.4 \%(w / v)$ tricaine at $48 \mathrm{hpf}$. The heart-beating rate was measured over 3 min using a microscope (Olympus, Japan), and results are represented as the average heart-beating rate per min (Lee et al. 2013). Cell death was detected in live embryos using acridine orange staining at $72 \mathrm{hpf}$ (Kang et al. 2013). For teratotoxicity assay, after zebrafish embryos exposed to SCDE for 24 and $48 \mathrm{hpf}$, non-lethal malformations were observed under the microscope (Leica, Leica Microsystems, Bannockburn, IL). All experiments were carried out in triplicates.

Evaluation of cell death and generation of intracellular reactive oxygen species (ROS) and nitric oxide (NO) in lipopolysaccharide (LPS)-stimulated zebrafish embryo Synchronized zebrafish embryos were collected and arrayed by a pipette in 12-well plates. Each well contained $2 \mathrm{ml}$ of the embryo medium with 15 embryos during 7-9 hpf. Then, the embryos were incubated with or without SCDE for $1 \mathrm{~h}$. To induce inflammation, the embryos were exposed to $10 \mu \mathrm{g} / \mathrm{ml}$ LPS dissolved in the embryo medium for $24 \mathrm{hpf}$ at $28.5^{\circ} \mathrm{C}$. Thereafter, zebrafish embryos were transferred into fresh embryo medium, where they developed for up to $72 \mathrm{hpf}$. Cell death and intracellular ROS and NO generation in zebrafish embryos were estimated according to previously reported methods (Kang et al. 2013; Lee et al. 2013).

Cell death was detected in live embryos using acridine orange staining, a nucleic acid selective metachromatic dye that interacts with DNA and RNA by intercalation or electrostatic attractions. Acridine orange stain cells with disturbed plasma membrane permeability, so it preferentially stains necrotic or very late apoptotic cells. The zebrafish embryos were transferred into 24-well plates and treated with acridine orange solution $(7 \mu \mathrm{g} /$ $\mathrm{ml}$ ), and the plates were incubated for $30 \mathrm{~min}$ in the dark at $28.5{ }^{\circ} \mathrm{C}$. After incubation, the embryos were rinsed with fresh embryo media and anesthetized before observation and observed under a fluorescence microscope, which was equipped with a CoolSNAP-Pro color digital camera (Olympus, Tokyo, Japan). The images of stained embryos were analyzed for cell death, and fluorescence intensity of individual embryos was quantified using ImageJ 1.46r software (Wayne Rasband, National Institutes of Health, Bethesda, MD, USA). Cell death were calculated by comparing the fluorescence intensity of treatment embryos to the controls. 
Generation of ROS in zebrafish embryos was analyzed using an oxidation-sensitive fluorescent probe dye, $2^{\prime}, 7^{\prime}$ dichlorodihydrofluorescein diacetate (DCF-DA). The zebrafish embryos were transferred into 24-well plates and treated with DCF-DA solution $(20 \mu \mathrm{g} / \mathrm{ml})$, and the plates were incubated for $1 \mathrm{~h}$ in the dark at $28.5{ }^{\circ} \mathrm{C}$. After incubation, the embryos were rinsed with fresh embryo media and anesthetized before observation and observed under a fluorescence microscope, which was equipped with a CoolSNAP-Pro color digital camera (Olympus, Tokyo, Japan). The images of stained embryos were analyzed for ROS generation, and fluorescence intensity of individual embryos was quantified using Image J 1.46r software (Wayne Rasband, National Institutes of Health, Bethesda, MD, USA). Generation of ROS were calculated by comparing the fluorescence intensity of treatment embryos to the controls.

Generation of NO in zebrafish embryos was analyzed using a fluorescent probe dye, diamino-fluorophore 4amino-5-methylamino-2', $7^{\prime}$-difluorofluorescein diacetate (DAF-FM DA). The zebrafish embryos were transferred into 24-well plates and treated with DAF-FM DA solution $(5 \mu \mathrm{M})$ and incubated for $1 \mathrm{~h}$ in the dark at $28.5^{\circ} \mathrm{C}$. After incubation, the embryos were rinsed with fresh embryo media and anesthetized before observation and observed under a fluorescence microscope, which was equipped with a CoolSNAP-Pro color digital camera (Olympus, Tokyo, Japan). The images of stained embryos were analyzed for NO generation, and fluorescence intensity of individual embryos was quantified using Image 1.46r software (Wayne Rasband, National Institutes of Health, Bethesda, MD, USA). Generation of NO were calculated by comparing the fluorescence intensity of treatment embryos to the controls.

\section{Statistical analysis}

The data are presented as means \pm standard error (SE). Statistical comparisons of the mean values were performed by analysis of variance (ANOVA), followed by Duncan's multiple range test using SPSS software. Statistical significance was considered at $P<0.05$.

\section{Results}

\section{Effect of SCDE on survival rate, heart beat rate, morphological} changes, and cell death in zebrafish embryo

In order to determine the toxicity of the SCDE, in this study, we observed the survival rate, heart beat rate, and morphological changes in zebrafish embryos after exposure to different concentrations of SCDE. As shown Fig. 1a, 1, 10, and $100 \mu \mathrm{g} / \mathrm{ml}$ of SCDE did not significantly caused zebrafish embryo death throughout assay. Mortality was caused after exposure to 200 and $400 \mu \mathrm{g} /$ $\mathrm{ml}$ of SCDE at $24 \mathrm{hpf}$, respectively (Fig. 1a). Especially, $400 \mu \mathrm{g} / \mathrm{ml}$ of SCDE caused approximately $90 \%$ embryo mortality since $24 \mathrm{hpf}$. We did not investigate the zebrafish embryo of 200 and $400 \mu \mathrm{g} / \mathrm{ml}$ for further analyses due to the lethal toxicity was too high. In the heart beat rate test, there is no significant change in heart beat rate compared to the control indicating that there is no toxicity at the tested concentrations (Fig. 1b).

To examine morphologic defects caused by SCDE, developmental abnormalities of zebrafish embryos exposed to SCDE were analyzed at 24 and $48 \mathrm{hpf}$. As shown Fig. 1c, no morphological abnormalities in zebrafish embryos were observed at the tested concentrations of SCDE, which indicated that SCDE did not have any toxic effects on the developmental stages of zebrafish embryos.

To evaluate whether SCDE has toxic effect to the cells, zebrafish embryos were treated with SCDE for $72 \mathrm{~h}$, and cell death was measured via acridine orange staining assay. SCDE was not exerting any cytotoxic effect at the indicated concentrations $(25,50$, and $100 \mu \mathrm{g} / \mathrm{ml})$ in zebrafish embryos (Fig. 1d). With the results of the preliminary studies, we selected the SCDE concentrations as 25,50 , and $100 \mu \mathrm{g} / \mathrm{ml}$ for further experiments.

\section{In vivo effect of SCDE on LPS-induced ROS generation}

We investigated LPS-induced ROS generation in zebrafish embryos using oxidation sensitive fluorescent probe dye, DCF-DA. Figure 2 shows the protective effect of SCDE on LPS-induced ROS generation. The control, which contained no LPS or SCDE, generated clear image, whereas treated only with LPS, generated fluorescence image, which suggests that generation of ROS has taken place in the presence of LPS in the zebrafish embryos. However, when the zebrafish embryos were treated with SCDE prior to LPS treatment; a dose-dependent reduction in the generation of ROS was observed.

\section{In vivo effect of SCDE B on LPS-induced NO production} The effect of SCDE on LPS-induced NO production was shown in Fig. 3. Stimulation of the zebrafish embryos with LPS resulted in an enhancement of NO production. However, pretreatment of zebrafish embryos with the SCDE decreased the NO production in a dose-dependent manner.

In vivo protective effect of SCDE on LPS-induced cell death The protective effect of SCDE on LPS-induced cell death was shown in Fig. 4. Cell death in zebrafish embryos was significantly elevated by the LPS treatment as compared with non-LPS-treated zebrafish embryos. However, the LPS-induced cell death in SCDE-treated zebrafish embryos was significantly reduced in a dosedependent manner. 

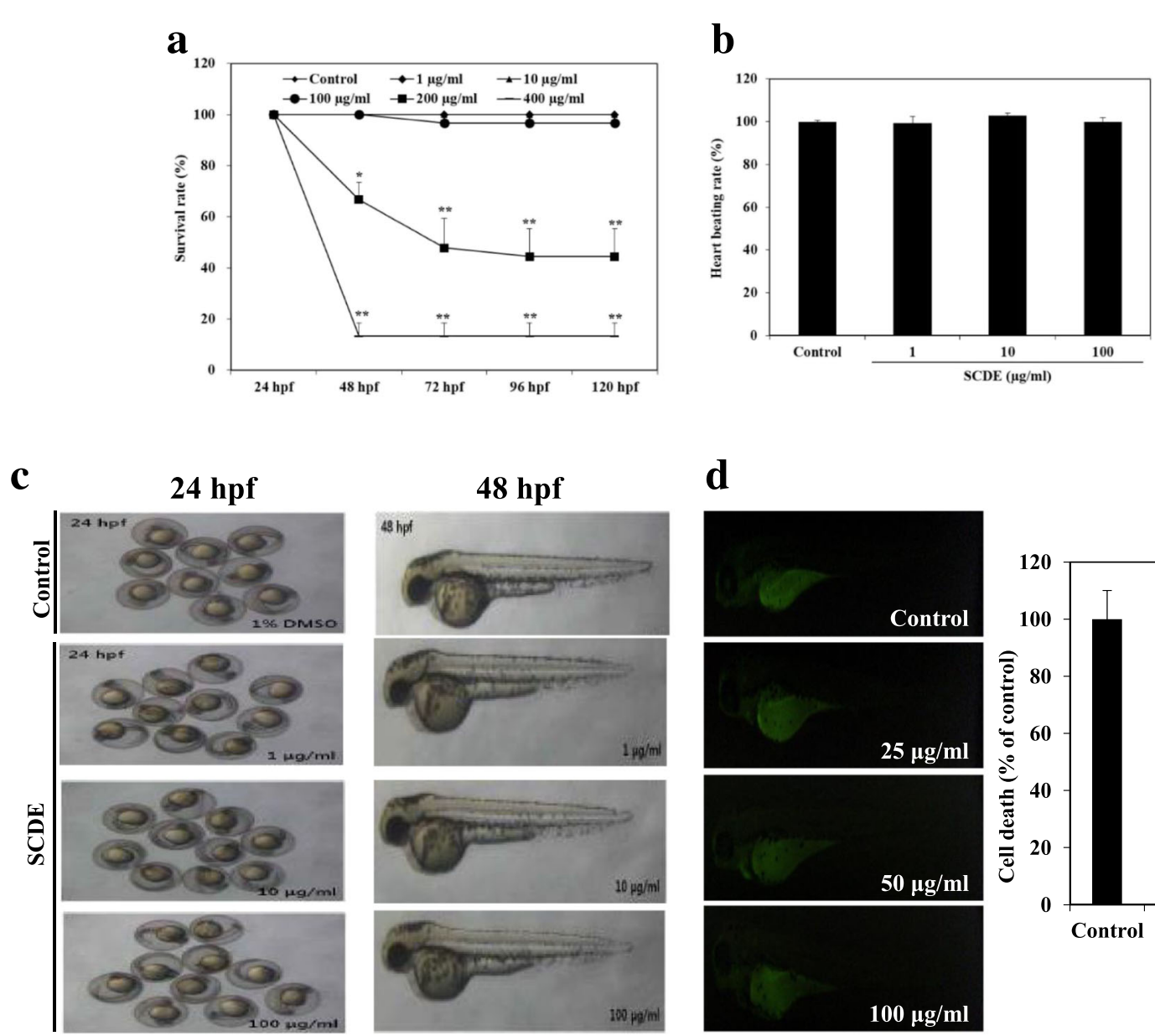

Fig. 1 Developmental toxicity of SCDE in zebrafish embryos. a Survival rates throughout 24-120 hpf. b Heart beating rates at 48 hpf. The values are expressed as the mean \pm SE. Significant differences from the untreated group were identified at $* P 0.05$ and ${ }^{* *} P<0.01$. c Representative images of developmental malformations in zebrafish embryos exposed to indicated concentration of SCDE at 24 and 48 hpf. $\mathbf{d}$ Effect of SCDE on cell death in zebrafish embryos. The cell death levels were measured after acridine orange staining by image analysis and fluorescence microscope. Representative fluorescence micrographs of cell death level, and data obtained from individual zebrafish fluorescence intensity analyses using an image J program are shown as bar graph. The values are expressed as the mean \pm SE

\section{Discussion}

Several studies revealed anti-inflammatory candidates found in soft corals (Fenical 1987; Radhika et al. 2005; $\mathrm{Hu}$ et al. 2011). However, there is a lack of information regarding the anti-inflammatory activities and toxicity of soft corals in vivo model. Recent studies have reported that zebrafish was used to rapidly and simply assess the anti-inflammatory activity against LPS-stimulated inflammation and toxicity (Park and Cho 2011; He et al. 2013). Hence, the aim of the present investigation was to evaluate the toxicity and anti-inflammatory effect of the soft coral D. gigantea extract (SCDE) in the zebrafish embryo in vivo model.

A significant reduction in survival rate following exposure to 200 and $400 \mu \mathrm{g} / \mathrm{ml}$ of SCDE at $24 \mathrm{hpf}$ was observed in our study. However, 1,10 , and $100 \mu \mathrm{g} / \mathrm{ml}$ of SCDE did not significantly caused zebrafish embryo's death. Therefore, we did not investigate the zebrafish embryo of 200 and $400 \mu \mathrm{g} / \mathrm{ml}$ for further analyses due to the lethal toxicity was too high. The heart is one of the first functional organ developed in zebrafish, and heart beat rate is an important toxicology end point in the embryonic test, so measurement of heart beat rate is also an important variable of interest in assessing cardiac toxicity (De et al. 2014). Our results showed that exposure to SCDE did not affect the heart beat rate of zebrafish embryo; there was no significant change compared to the control indicating that there is no toxicity at the tested concentrations. Based on the toxicological outcomes obtained from our study, we explored the developmental toxicity of SCDE by observation of morphologic defects in zebrafish embryo. Morphological alterations usually follow molecular and biochemical changes in toxicity syndromes (Liu et al. 2015). Our 

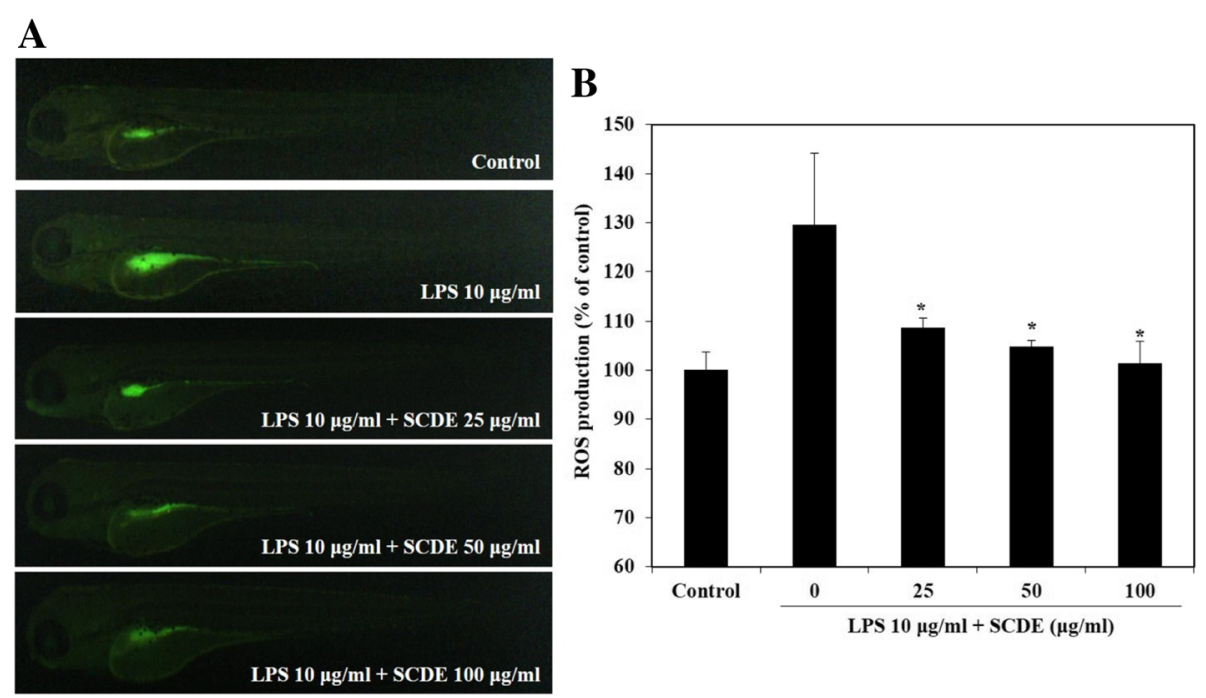

Fig. 2 Inhibitory effect of SCDE on LPS-stimulated ROS production in zebrafish embryos. The ROS levels were measured after staining with DCF-DA by image analysis and fluorescence microscope. a Representative fluorescence micrographs of ROS production and $\mathbf{b}$ data obtained from individual zebrafish fluorescence intensity analyses using an image J program are shown as bar graph. The values are expressed as the mean \pm SE. Significant differences from the only LPS-treated group were identified at ${ }^{*} P<0.05$

results showed that no morphological abnormalities in zebrafish embryos were observed at the tested concentrations of SCDE. These results clearly show that SCDE did not have any toxic effects on the developmental stages of zebrafish embryos.

A high ROS level induces oxidative stress which can result in the development of a variety of cell or tissue injury associated with degenerative diseases including inflammation. Our results showed that treating zebrafish embryos with LPS treatment significantly increased ROS level. However, SCDE inhibited this LPS treatment-induced ROS generation. These results demonstrate that SCDE alleviated inflammation by inhibiting the ROS generation induced by LPS treatment. NO is an important inflammatory mediator that is synthesized from arginine by nitric oxide synthase (NOS). Under pathological condition, NO production is increased by the inducible NOS (iNOS), subsequently brings about cytotoxicity and tissue damage
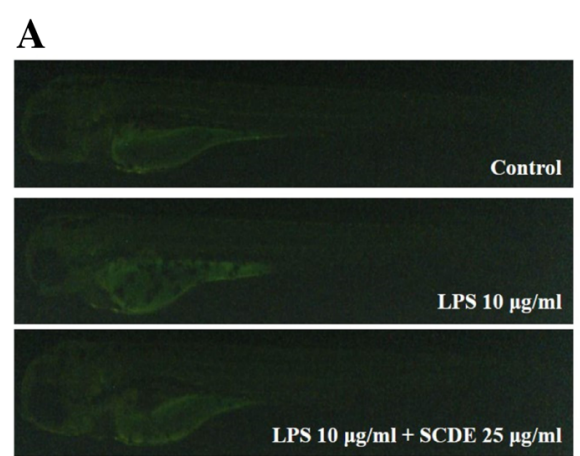

B

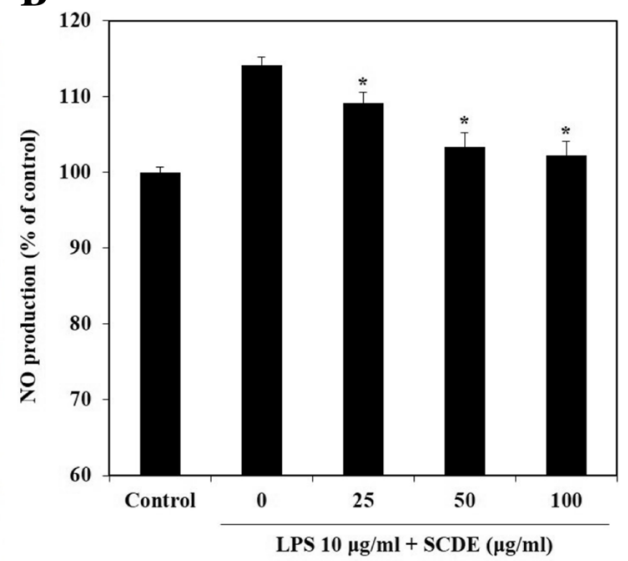

LPS $10 \mu \mathrm{g} / \mathrm{ml}+$ SCDE $100 \mu \mathrm{g} / \mathrm{ml}$

Fig. 3 Inhibitory effect of SCDE on LPS-stimulated NO production in zebrafish embryos. The NO levels were measured after staining with DAF-FM-DA by image analysis and fluorescence microscope. $\mathbf{a}$ Representative fluorescence micrographs of NO production, and $\mathbf{b}$ data obtained from individual zebrafish fluorescence intensity analyses using an image J program are shown as bar graph. The values are expressed as the mean \pm SE. Significant differences from the only LPS-treated group were identified at ${ }^{*} P<0.05$ 


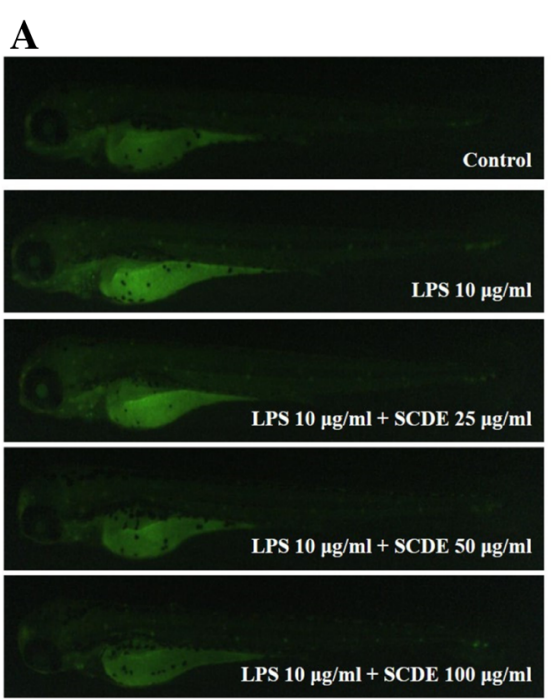

B

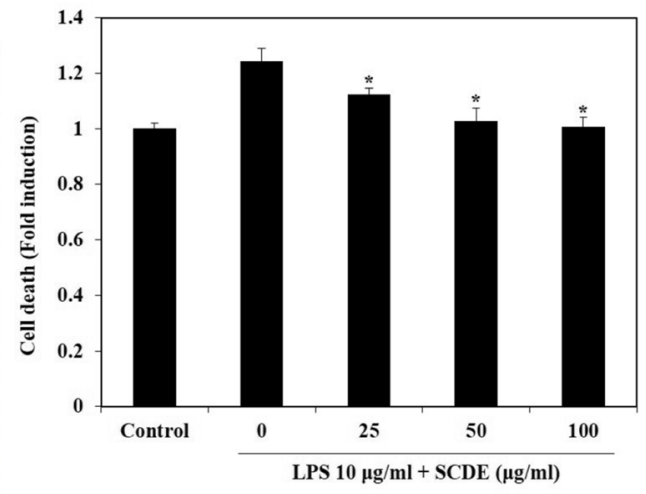

Fig. 4 Protective effect of SCDE on LPS-stimulated cell death in zebrafish embryos. The cell death levels were measured after staining with acridine orange by image analysis and fluorescence microscope. a Representative fluorescence micrographs of cell death level and $\mathbf{b}$ data obtained from individual zebrafish fluorescence intensity analyses using an image J program are shown as bar graph. The values are expressed as the mean \pm SE. Significant differences from the only LPS-treated group were identified at ${ }^{*} P<0.05$

(Kim et al. 1999). Therefore, NO inhibitors are essential for the prevention of inflammatory diseases. Previous studies have indicated that extract of $D$. gigantea suppressed NO generation and the expression of iNOS and proinflammatory cytokines in murine macrophage cells (Wang et al. 2016). SCDE in this study also significantly reduced the elevated NO level induced by LPS treatment in zebrafish embryos. Although the expression level of iNOS and proinflammatory cytokines were not examined, SCDE may therefore inhibit iNOS and proinflammatory cytokines expression in zebrafish based on previously published data. These findings indicate that SCDE might confer important protection against the inflammation induced by chemical damage. Such cellular damage frequently impairs the metabolic function and results in cell death (Finkel and Holbrook 2000). In the present study, we found that SCDE protected against LPS-induced cytotoxic effects in zebrafish embryos. These findings suggest that SCDE might confer important protection against the cellular damage induced by oxidative stress.

Overall, the above results suggest that SCDE could act as strong inhibitors of ROS and NO in LPS-stimulated inflammatory zebrafish model. In addition, SCDE has a protective effect against the cell damage induced by LPS exposure in zebrafish embryos. This outcome could explain the potential anti-inflammatory activity of SCDE, which might have a beneficial effect during the treatment of inflammatory diseases.

\section{Conclusions}

In conclusion, the direct effects of SCDE on antiinflammatory activities in vivo model remained to be determined. Therefore, in the present study, we investigated the anti-inflammatory effect of SCDE, on LPSstimulated inflammation, in in vivo zebrafish model. As expected, SCDE demonstrated strong anti-inflammatory properties against LPS treatment-induced inflammation. The SCDE also did not have any toxic effects in zebrafish embryos. The SCDE exhibited profound antiinflammatory effect both in vitro as well as in vivo, suggesting that the SCDE might be a strong antiinflammatory agent. Furthermore, further studies are needed to explore to identify them as the major components in the SCDE.

\section{Abbreviations}

DAF-FM DA: Diamino-fluorophore 4-amino-5-methylamino-2',7'-difluorofluorescein diacetate; DCF-DA: 2',7'-Dichlorodihydrofluorescein diacetate; LPS: Lipopolysaccharide; NO: Nitric oxide; ROS: Reactive

oxygen species; SCDE: Soft coral Dendronephthya gigantea extract

\section{Funding}

This research was financially supported by the Ministry of Trade, Industry, and Energy (MOTIE), Korea, under the "Regional Specialized Industry Development Program" supervised by the Korea Institute for Advancement of Technology (KIAT).

Availability of data and materials Not applicable.

Ethics approval and consent to participate Not applicable.

Consent for publication

Not applicable.

Competing interests

The authors declare that they have no competing interests. 


\section{Publisher's Note}

Springer Nature remains neutral with regard to jurisdictional claims in published maps and institutional affiliations.

Received: 1 November 2017 Accepted: 27 November 2017

Published online: 18 December 2017

\section{References}

Ali S, Champagne DL, Spaink HP, Richardson MK. Zebrafish embryos and larvae: a new generation of disease models and drug screens. Birth Defects Res C Embryo Today. 2011;93:115-33.

Blunt JW, Copp BR, Keyzers RA, Munroa MHG, Prinsep MR. Marine natural products Nat Prod Rep. 2012;29:144-222.

Chao CH, Wen ZH, Chen IM, Su JH, Huang HC, Chiang MY, Sheu JH. Anti-inflammatory steroids from the octocoral Dendronephthya griffin. Tetrahedron. 2008:64:3554-60.

Cho IY, Kang DW, Kang J, Hwang H, Won JH, Paek WK, Seo SY. A study on the biodiversity of benthic invertebrates in the waters of Seogwipo, Jeju Island, Korea. J Asia Pac Biodivers. 2014;7:e11-8.

De LE, Zaccaria GM, Hadhoud M, Rizzo G, Ponzini R, Morbiducci U, Santoro MM ZebraBeat: a flexible platform for the analysis of the cardiac rate in zebrafish embryos. Sci Rep. 2014;4:649-52.

den Hertog J. Chemical genetics: drug screens in zebrafish. Biosci Rep. 2005:25: 289-97.

Eisen JS. Zebrafish make a big splash. Cell. 1996:87:969-77.

Elkhayat ES, Ibrahim SRM, Fouad MA, Mohamed GA. Dendronephthols A-C, new sesquiterpenoids from the Red Sea soft coral Dendronephthya sp. Tetrahedron. 2014;70:3822-5

Fenical W. Marine soft corals of the genus Pseudopterogorgia: a resource for novel anti-inflammatory diterpenoids. J Nat Prod. 1987;50:1001-8.

Finkel TN, Holbrook J. Oxidants, oxidative stress and the biology of aging. Nature. 2000:408:239-47.

Fishman MC. Zebrafish genetics: the enigma of arrival. Proc Natl Acad Sci U S A. 1999:96:10554-6.

He JH, Guo SY, Zhu F, Zhu JJ, Chen YX, Huang CJ, Gao JM, Dong QX, Xuan YX, Li CQ. A zebrafish phenotypic assay for assessing drug-induced hepatotoxicity. J Pharmacol Toxicol Methods. 2013;67:25-32.

Hu J, Yang B, Lin X, Zhou X, Yang X, Long L, Liu Y. Chemical and biological studies of soft corals of the Nephtheidae family. Chem Biodivers. 2011;8:1011-32.

Kang MC, Cha SH, Wijesinghe WAJP, Kang SM, Lee SH, Kim EA, Song CB, Jeon YJ. Protective effect of marine algae phlorotannins against AAPH-induced oxidative stress in zebrafish embryo. Food Chem. 2013;138:950-5.

Kim HK, Cheon BS, Kim YH, Kim SY, Kim HP. Effects of naturally occurring flavonoids on nitric oxide production in the macrophage cell line RAW 264.7 and their structure-activity relationships. Biochem Pharmacol. 1999;58:759-65.

Lakshmi V, Kumar R. Metabolites from Sinularia species. Nat Prod Res. 2009;23: 801-50.

Lee $\mathrm{SH}, \mathrm{Ko} \mathrm{Cl}$, Jee $\mathrm{Y}$, Jeong Y, Kim M, Kim JS, Jeon YJ. Anti-inflammatory effect of fucoidan extracted from Ecklonia cava in zebrafish model. Carbohydr Polym. 2013;92:84-9.

Li G, Deng Z, Guan H, van Ofwegen L, Proksch P, Lin W. Steroids from the soft coral Dendronephthya sp. Steroids. 2005;70:13-8.

Liao YF, Chiou MC, Tsai Wen CC, Wang YH, Cheng CC, Chen YH. Resveratrol treatment attenuates the wound-induced inflammation in zebrafish larvae through the suppression of myeloperoxidase expression. J Food Drug Anal. 2011:19:167-73.

Liu H, Gooneratne R, Huang X, Lai R, Wei J, Wang W. A rapid in vivo zebrafish model to elucidate oxidative stress-mediated PCB126-induced apoptosis and developmental toxicity. Free Radic Biol Med. 2015;84:91-102.

Park $\mathrm{KH}$, Cho $\mathrm{KH}$. A zebrafish model for the rapid evaluation of pro-oxidative and inflammatory death by lipopolysaccharide, oxidized low-density lipoproteins, and glycated high-density lipoproteins. Fish Shellfish Immunol. 2011:31:904-10.

Radhika P, Rao PR, Archana J, Rao NK. Anti-inflammatory activity of a new sphingosine derivative and cembrenoid diterpene (lobohedleolide) isolated from marine soft corals of Sinularia crassa Tixier-Durivault and Lobophytum species of the Andaman and Nicobar Islands. Biol Pharm Bull. 2005:28:1311-3.

Tomono Y, Hirota H, Imahara Y, Fusetani NJ. Four new steroids from two octocorals. J Nat Prod. 1999;62:1538-41.

Wang L, Oh JY, Shanura Fernando IP, Asanka Sanjeewa KK, Kim EA, Lee WW, Jeon YJ. Soft corals collected from Jeju Island; a potential source of anti-inflammatory phytochemicals. J Chitin Chitosan. 2016:21:247-54.

\section{Submit your next manuscript to BioMed Central and we will help you at every step:}

- We accept pre-submission inquiries

- Our selector tool helps you to find the most relevant journal

- We provide round the clock customer support

- Convenient online submission

- Thorough peer review

- Inclusion in PubMed and all major indexing services

- Maximum visibility for your research

Submit your manuscript at www.biomedcentral.com/submit 